\title{
A Hybrid Method for Underwater Image Preprocessing
}

\author{
Hengxu $\mathrm{Ma}^{1}$, Wang Kun ${ }^{1}$
}

${ }^{1}$ College of Instrument \& Meter Engineering, Chengdu University of Technology, Chengdu 610059, China

\begin{abstract}
This paper provides a hybrid method for underwater image restoration. Firstly, for the color distortion caused by different light attenuation characteristics, the automatic color balance algorithm which based on dynamic threshold is used to correct the color of underwater images. Secondly, the image dehazing based on the dark primary color principle is adopted, which transmissivity and background light are modified according to the propagation characteristics of underwater light. Finally, aiming at the over exposure of underwater images after dehazing, the gray world light compensation algorithm is employed to improve the visual display of underwater images. Experiments have shown the effectiveness of the system designed in this paper.
\end{abstract}

Keywords: Underwater Image, Color Distortion Correction, Dehazing Algorithm, Exposure Compensation

\section{Introduction}

The complex imaging environment makes underwater images generally have the following characteristics: Because of the different attenuation of underwater light at different wavelengths, the underwater image has the problem of color loss; The backscattering of water by light causes the image to be blurred which makes the image seem to be covered with mist. In addition, the suspended particles in water make the image very noisy. These features bring challenges and difficulties to underwater image processing. In recent decades, underwater image preprocessing has attracted more and more attention.

The image dehazing algorithm is similar to the underwater image imaging model, so it is adapted to the underwater image sharpening method. For example, both the image atomization imaging model and the underwater image imaging model are caused by the serious light scattering degradation because scattering medium absorbs and scatters light (a large number of small particles contained in the mist and fine particles in the water), which causes severe image atomization, contrast and a loss of image detail. In the literature [2], the possibility about the dehazing algorithm use to underwater image enhancement is mentioned. By comparing the differences between underwater images and foggy images, after the improvement of the dehazing algorithm, it can be applied to underwater image enhancement processing.

Firstly, aiming at the color distortion caused by the attenuation characteristics of different light, the underwater image is corrected by the automatic white balance algorithm which based on dynamic threshold. Secondly, through the image dahazing technology based on dark primary color principle, and transmissivity estimation model and background light estimation have been corrected which aimed at underwater propagation characteristics, the dark primary channel image enhancement algorithm which apply to underwater is proposed. Finally, for the overexposure problem of the underwater image after dehazing, the gray world ray compensation algorithm is used to restore the visual display of underwater image. The experiment has verified the effectiveness of the system designed in this paper.

\section{The hybrid method}

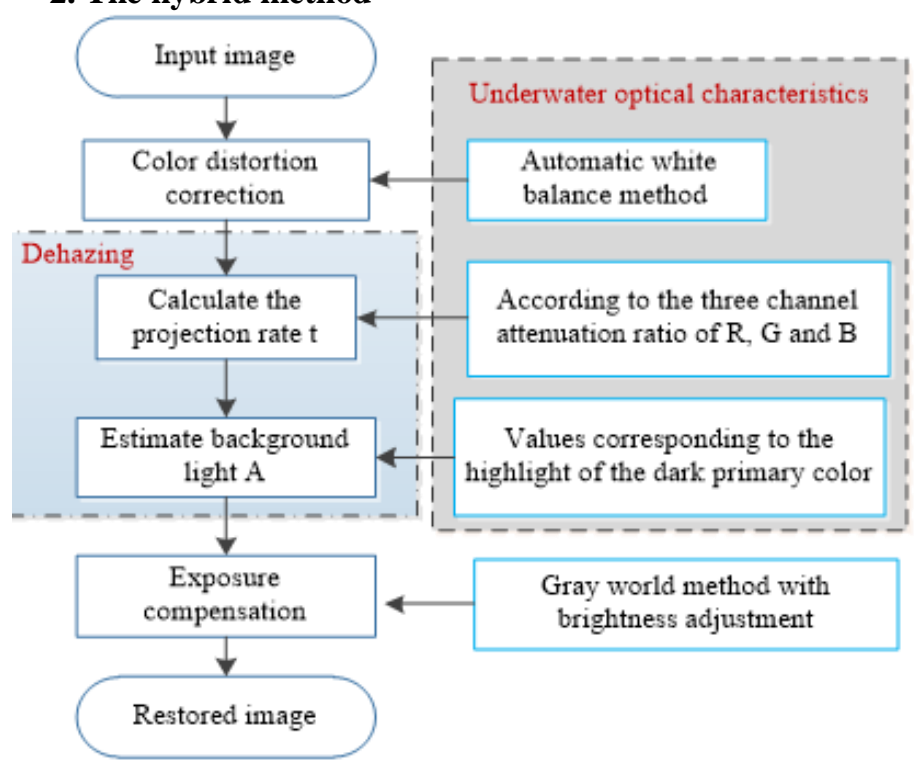

Fig.1 Algorithm design process

After gaining the original image, the underwater image color distortion correction is performed first, the 
automatic white balance method based on the dynamic threshold is used to correct the color distortion of the underwater image with blue (green) keynote. Then do the relatively calculation of the dehazing algorithm (calculate the transmissivity $t$ and estimate the underwater background light $\mathrm{A}$ ), perform the exposure compensation after dehazing enhancement so that the image would not appear a high overall brightness. Finally, driving out the restored image.

\subsection{The correction method of underwater image color distortion}

Due to the difference in the imaging model of the underwater image, the color of underwater image is different, and the attenuation and the different scatter coefficient cause the overall image blue (green). This paper introduces an automatic white balance method based on dynamic threshold to correct the color distortion of underwater images.
The automatic white balance algorithm is usually divided into two steps: the detection and adjustment of white points. The automatic white balance method used in this paper is based on a dynamic threshold to detect white points, so that it is called the automatic white balance method based on the dynamic threshold. In the practical operation of the algorithm, the image is divided into regions at first, which divide a whole image into a number of small parts. Then the reference white point is gained by decomposition calculation in each part. The next step is to use the Von Thunen model to calculate the image and then adjust the image to achieve a white balance effect and correct the color distortion of the underwater image.

\section{2 underwater image dehazing image processing based on dark primary color principle}

The general flow chart of the dehazing algorithm is shown in Figure 2.

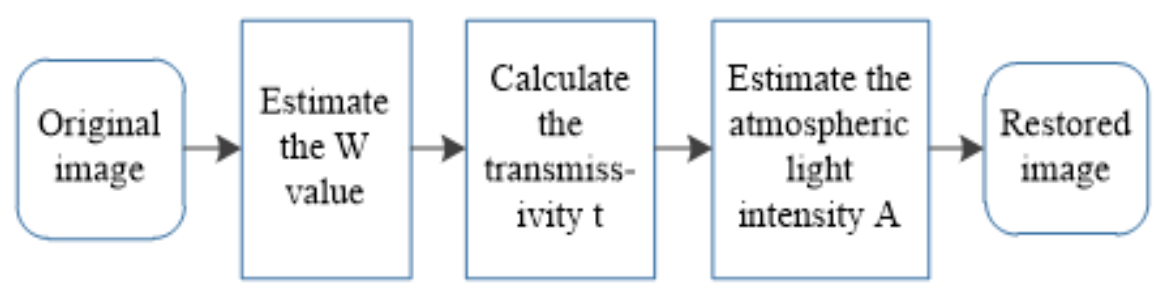

Fig.2 Flowchart of dehazing algorithm

In the formula, the $\mathrm{I}(\mathrm{x})$ is the received image (the image to be processed), $\mathrm{J}(\mathrm{x})$ is the image to be restored (the image after dehazing), the A represents the background atmospheric light component, $t(x)$ is used to express the portion (transmissivity) that the light penetrates the transmission medium to the vision facility without attenuation. The image enhance dehazing is the recovery of a fog-free image from a foggy image.

(1)Determination value the value of $\omega$

It is necessary to retain a certain amount of fog during the dehazing process. At this time, we need to introduce a transmission coefficient $\omega$ to ensure the image that after dehazing can leave a little fog to reflect the depth of field.

(2) Transmissivity estimation

The estimation of transmissivity $\tilde{t}(x)$ :

By introducing that requires a little fog, a factorwbetween $[0,1]$ is introduced to equation (3.9), and equation (3.9) is corrected to:

$\tilde{t}(x)=1-\omega \min _{C}\left(\min _{C} \frac{I^{c}(y)}{A^{c}}\right)$

The transmissivity of the dark primary color algorithm which was mentioned earlier cannot apply to the process of underwater images directly. That is because there is a problem in underwater images that the attenuation of the $\mathrm{R}$ channel is the most serious. Therefore, the transmissivity also needs to be corrected.
The relationship between the scattering coefficient of water and the wavelength of light is obtained firstly after a fine-tuning the transmissivity by the ratio of the scattering coefficient of light under water:

$b_{\lambda}=(-0.00113 \lambda+1.62517) b\left(\lambda_{r}\right)$

The $b\left(\lambda_{\gamma}\right)$ expresses the scattering coefficient of the reference wavelength.

The transmissivity obtained above regard as the transmissivity of $\mathrm{R}$ channel. Then giving a fine tuning to transmissivity of channel $\mathrm{G}$ and channel $\mathrm{B}$ by the ratio $b_{G} / b_{R}$ and $b_{B} / b_{R}$ of the scattering coefficient ( because the transmissivity ratio is a ratio, the above $b\left(\lambda_{\gamma}\right)$ in formula will be canceled out). The fine-tuned transmissivities are applied to each of the three RGB channels.

(3) background light estimation

The specific steps to obtain the background atmospheric light A value are as follows:

(1) From the dark channel image (the gray-scale image), arranges the pixels from large to small according to the brightness and extracts the first $0.5 \%$ pixels.

(2)

(3) Among these pixel points, the value of corresponding point with the highest brightness which is fund in original foggy image I is regarded as A value. 
Hereinbefore, all above assume that the background light is estimated by atmospheric light estimation, but the background light of underwater image is different from the atmospheric light, so it is necessary to estimate of RGB three-way channel individually, and find the value of the point with this brightness from the brightest point in dark channel of RGB three-way channel to the three channels of RGB of original image.

\section{3 light compensation}

After dehazing, the image effect will appear overexposure phenomenon, so the image after dehazing needs further light compensation processing.

(1) Grayscale world method

The grayscale world algorithm is based on the "grayscale world hypothesis", which holds that: for an image with a large number of color changes, the average value of the three color components of its RGB is close to the same grayscale value. The basic idea of this algorithm is: firstly, calculate the average value of the three color components of RGB and the average gray value of the original image, and then adjust the value of the three components of RGB for each pixel, so that the average value of the three color components after the adjustment is close to the average gray value.

(2) Grayscale world method for brightness adjustment

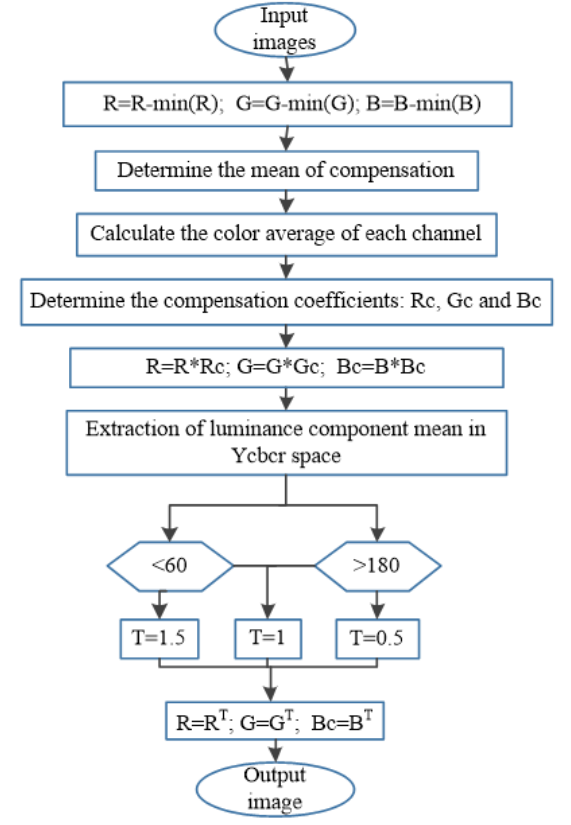

Fig.3 Light compensation

The image adjusted by the grayscale world algorithm, the light adjustment effect is less obvious, and the influence of uneven brightness still exists. Therefore, it is necessary to consider that turn the image after the grayscale world compensation to Ycbcr space and extract the brightness $\mathrm{Y}$ space value is extracted, then adjust the coefficient more according to this value.

\section{Experiments}
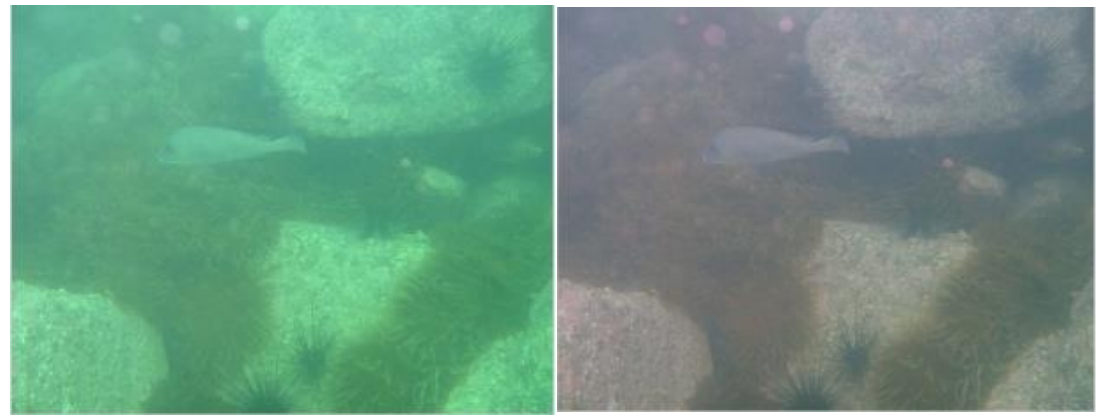

(a) Original foggy underwater image

(b) Image after white balance

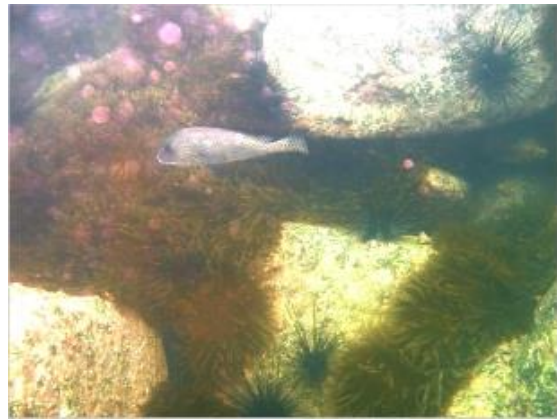

(c) Image after dehazing

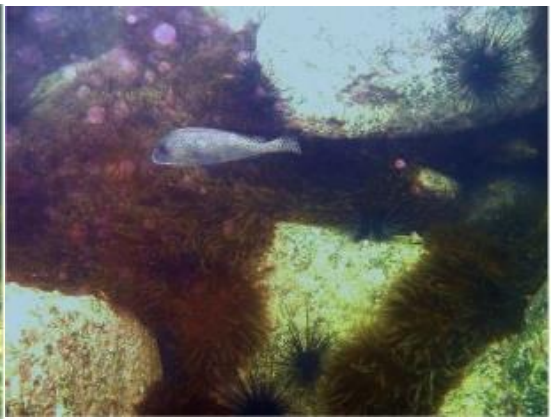

(d) Image after light compensation

Fig.4 The effect image of underwater image dehazing processing 
Figure 4 is a typical underwater image with a bluish (green) color keynote. After the automatic white balance processing, the color distortion can be effectively corrected. Then through the dehazing algorithm processing and light compensation, the enhanced image after dehazing can be obtained. Underwater image generally requires a auxiliary to shoot with artificial light source, so the brightness of the underwater background light will be overall (after light compensation there is still have problems with brightness is larger), further affect the underwater background light estimation. It is necessary to find a more advanced underwater background light estimation algorithm for the underwater image. It will be the main content of the research and work in the next stage.

\section{Conclusions}

In this paper, the dark channel prior dehazing algorithm is used to carry out underwater image enhancement, and the underwater image enhancement based on the dark channel prior dehazing algorithm is improved, which makes the original dehazing algorithm that deal with the atmospheric light can used to deal a part of the underwater image which is non-blue (green) tone in the underwater image. Through experiments and simulation results, the dehazing algorithm based on dark channel prior can also directly process some underwater images with non-blue (green) tone, and the improved method that mainly based on blue (green) tone is more excellent for the underwater image. The automatic white balance algorithm based on dynamic threshold has solved the problem of large overall brightness after underwater image enhancement.

\section{References}

1. Zhang Yin. Underwater target image processing and shape recognition technology research [D], Heilongjiang, Harbin Engineering University, 2015

2. Yang Aiping, Zheng Jia, Wang Jian, He Yuqing. Underwater image restoration based on color distortion removal and dark channel prior $[\mathrm{J}]$. Journal of electronics and information technology, 2015, 37(11): 145-150 